\title{
An absence of neutrinos associated with cosmic-ray acceleration in $\gamma$-ray bursts
}

IceCube Collaboration*

Very energetic astrophysical events are required to accelerate cosmic rays to above $10^{18}$ electronvolts. GRBs ( $\gamma$-ray bursts) have been proposed as possible candidate sources ${ }^{1-3}$. In the GRB 'fireball' model, cosmic-ray acceleration should be accompanied by neutrinos produced in the decay of charged pions created in interactions between the high-energy cosmic-ray protons and $\gamma$-rays ${ }^{4}$. Previous searches for such neutrinos found none, but the constraints were weak because the sensitivity was at best approximately equal to the predicted flux ${ }^{5-7}$. Here we report an upper limit on the flux of energetic neutrinos associated with GRBs that is at least a factor of 3.7 below the predictions ${ }^{4,8-10}$. This implies either that GRBs are not the only sources of cosmic rays with energies exceeding $10^{18}$ electronvolts or that the efficiency of neutrino production is much lower than has been predicted.

Neutrinos from GRBs are produced in the decay of charged pions produced in interactions between high-energy protons and the intense $\gamma$-ray background within the GRB fireball, for example in the $\Delta$-resonance process $p+\gamma \rightarrow \Delta^{+} \rightarrow n+\pi^{+}$( $p$, proton; $\gamma$, photon (here $\gamma$-ray); $\Delta^{+}$, delta baryon; $n$, neutron; $\pi^{+}$, pion). When these pions decay via $\pi^{+} \rightarrow \mu^{+} v_{\mu}$ and $\mu^{+} \rightarrow e^{+} v_{e} \bar{v}_{\mu}$, they produce a flux of highenergy muon neutrinos $\left(v_{\mu}\right)$ and electron neutrinos $\left(v_{e}\right)$, coincident with the $\gamma$-rays, and peaking at energies of several hundred teraelectronvolts $(\mathrm{TeV})^{4,11}\left(\mu^{+}\right.$, antimuon; $e^{+}$, positron). Such a flux should be detectable using $\mathrm{km}^{3}$-scale instruments like the IceCube neutrino telescope $e^{12,13}$ (Supplementary Fig. 1). Owing to maximal mixing between muon neutrinos and tau neutrinos, neutrinos from pion decay in and around GRBs will arrive at Earth in an equal mixture of flavours. We focus here only on muons produced in $v_{\mu}$ charged-current interactions. As the downgoing cosmic-ray muon background presents challenges for the identification of neutrinoinduced muons, we achieve our highest sensitivity for upgoing neutrinos (from sources in the northern sky). However, the tight constraint of spatial and temporal coincidence with a GRB allows some sensitivity even in the southern sky. One of the two analyses presented here therefore includes Southern Hemisphere GRBs during the 200910 IceCube run.

The results presented here were obtained while IceCube was under construction, using 40 and 59 of the 86 photomultiplier strings of the final detector (Supplementary Fig. 1), which took data from April 2008 to May 2009 and from May 2009 until May 2010, respectively. During the 59-string data-taking period, 190 GRBs were observed and reported by $\gamma$-ray observatory satellites via the GRB Coordinates Network $^{14}$, with 105 in the northern sky. Of those GRBs, 9 were not included in our catalogue owing to detector downtime associated with construction and calibration. Two additional GRBs were included from test runs before the start of the official 59-string run. 117 northernsky GRBs were included from the 40 -string period ${ }^{7}$ to compute the final combined result. GRB positions were taken from the satellite with the smallest reported error, which is typically smaller than the IceCube resolution. The GRB $\gamma$-ray emission start $\left(T_{\text {start }}\right)$ and stop $\left(T_{\text {stop }}\right)$ times were taken by finding the earliest and latest time reported for $\gamma$-ray emission.
As in our previous study ${ }^{7}$, we conducted two analyses of the IceCube data. In a model-dependent search, we examine data during the period of $\gamma$-ray emission reported by any satellite for neutrinos with the energy spectrum predicted from the $\gamma$-ray spectra of individual $\mathrm{GRBs}^{6,9}$. The model-independent analysis searches more generically for neutrinos on wider timescales, up to the limit of sensitivity to small numbers of events at \pm 1 day, or with different spectra. Both analyses follow the methods used in our previous work ${ }^{7}$, with the exception of slightly changed event selection and the addition of the Southern Hemisphere to the model-independent search. Owing to the large background of downgoing muons from the southern sky, the Southern Hemisphere analysis is sensitive mainly to higher-energy events (Supplementary Fig. 3). Systematic uncertainties from detector effects have been included in the reported limits from both analyses, and were estimated by varying the simulated detector response and recomputing the limit, with the dominant factor being the efficiency of the detector's optical sensors.

In the 59-string portion of the model-dependent analysis, no events were found to be both on-source and on time (within $10^{\circ}$ of a GRB and between $T_{\text {start }}$ and $T_{\text {stop }}$ ). From the individual burst spectra ${ }^{6,9}$ with an assumed ratio of energy in protons to energy in electrons $\varepsilon_{p} / \varepsilon_{e}=10$ (ref. 6), 8.4 signal events were predicted from the combined 2-year data set and a final upper limit ( $90 \%$ confidence) of 0.27 times the predicted flux can be set (Fig. 1). This corresponds to a $90 \%$ upper limit on $\varepsilon_{p} / \varepsilon_{e}$ of 2.7 , with other parameters held fixed, and includes a $6 \%$ systematic uncertainty from detector effects.

In the model-independent analysis, two candidate events were observed at low significance, one $30 \mathrm{~s}$ after GRB 091026A (event 1) and another $14 \mathrm{~h}$ before GRB 091230A (most theories predict neutrinos within a few minutes of the burst). Subsequent examination showed they had both triggered several tanks in the IceTop surface air shower array, and are thus very probably muons from cosmic-ray air showers. In Fig. 2 are shown limits from this analysis on the normalization of generic power-law muon neutrino spectra expected from shock acceleration at Earth as a function of the size of the time window $|\Delta t|$, which is the difference between the neutrino arrival time and the first reported satellite trigger time. As a cross-check on both results, the limit from this analysis on the average individual burst spectra ${ }^{6,9}$ during the time window corresponding to the median duration of the bursts in the sample ( $28 \mathrm{~s}$ ) was 0.24 times the predicted flux, within $10 \%$ of the model-dependent analysis.

Assuming that the GRBs in our catalogue are a representative sample of a total of 667 per year (ref. 7), we can scale the emission from our catalogue to the emission of all GRBs. The resulting limits can then be compared to the expected neutrino rates from models that assume that GRBs are the main sources of ultra-high-energy cosmic rays $^{4,8,10}$, with sampling biases of the same order as model uncertainties in the flux predictions $s^{15,16}$. Limits from the model-independent analysis on fluxes of this type are shown in Fig. 3.

These limits exclude all tested models $s^{4,8-10}$ with their standard parameters and uncertainties on those parameters (Figs 1,3). The models are different formulations of the same fireball phenomenology,

* Lists of participants and their affiliations appear at the end of the paper. 


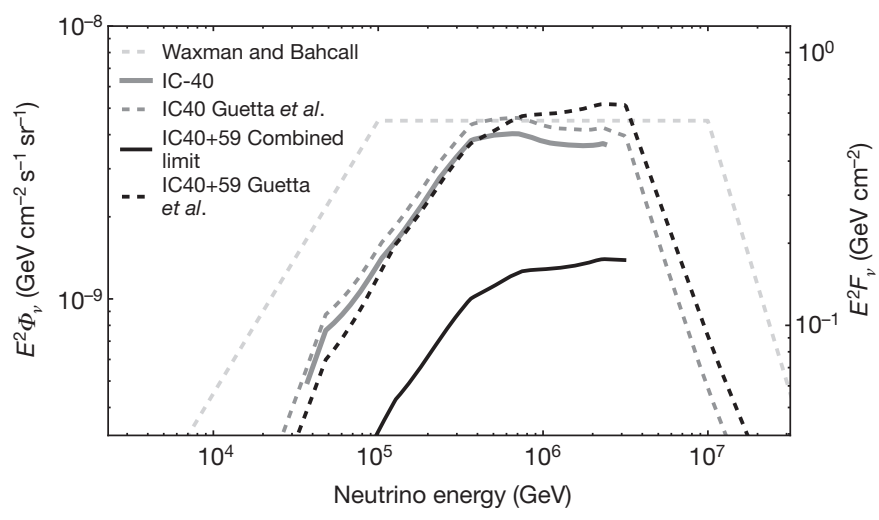

Figure 1 Comparison of results to predictions based on observed $\gamma$-ray spectra. The summed flux predictions normalized to $\gamma$-ray spectra ${ }^{6,9,19}$ are shown as a function of neutrino energy $(E)$ in dashed lines, with the dark grey dashed line labelled 'IC40 Guetta et al.' showing the flux prediction for the 40string portion of the analysis, and the black dashed line labelled 'IC40+59 Guetta et al.' showing the prediction for the full two-year dataset. The cosmic ray normalized Waxman-Bahcall flux ${ }^{4,20}$ is also shown for reference as the pale grey dashed line. $90 \%$ confidence upper limits on these spectra are shown as solid lines, with the grey line labelled 'IC40 limit' showing the previous IceCube result ${ }^{6}$ and the black 'IC40+IC59 Combined' line showing the result from the full dataset (this work). The predicted neutrino flux, when normalized to the $\gamma$-rays ${ }^{6,9}$, is proportional to the ratio of energy in protons to that in electrons, which are presumed responsible for the $\gamma$-ray emission $\left(\varepsilon_{p} / \varepsilon_{e}\right.$, here the standard 10 ). The flux shown is slightly modified ${ }^{6}$ from the original calculation ${ }^{9} . \Phi_{v}$ (left vertical axis) is the average neutrino flux at Earth, obtained by scaling the summed predictions from the bursts in our sample ( $F_{v}$, right vertical axis) by the global GRB rate (here $667{\text { bursts } \mathrm{yr}^{-1}}^{-1}$; ref. 7). The first break in the neutrino spectrum is related to the break in the photon spectrum measured by the satellites, and the threshold for photo-pion production, whereas the second break corresponds to the onset of synchrotron losses of muons and pions. Not all of the parameters used in the neutrino spectrum calculation are measurable from every burst. In such cases, benchmark values ${ }^{7}$ were used for the unmeasured parameters. Data shown here were taken from the result of the model-dependent analysis

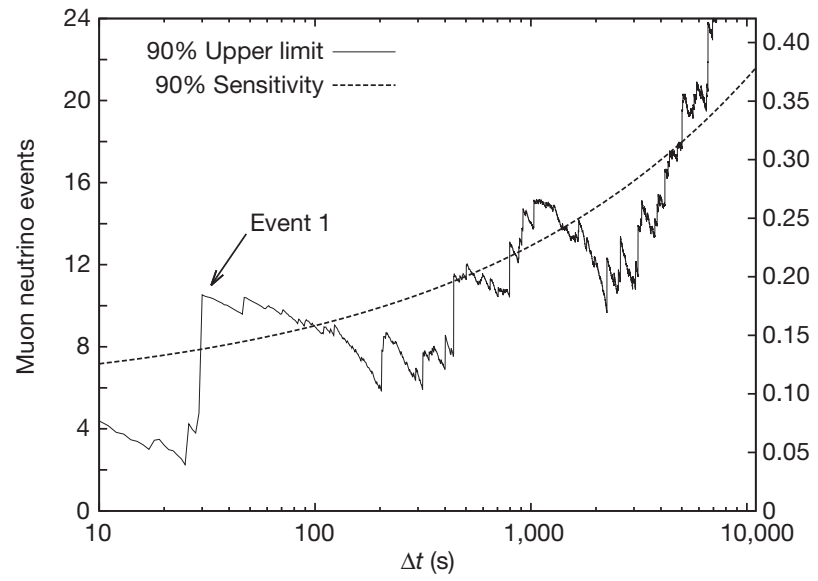

Figure 2 Upper limits on $E^{-2}$ power-law muon neutrino fluxes. Limits were calculated using the Feldman-Cousins method ${ }^{21}$ from the results of the model-independent analysis. The left-hand $y$-axis shows the total number of expected $v_{\mu}$ events, while the right-hand $y$-axis $\left(F_{v}\right)$ is the same as in Fig. 1. A time window of $\Delta t$ implies observed events arriving between $t$ seconds before the burst and $t$ afterward. The variation of the upper limit (solid line labelled ' $90 \%$ Upper limit') with $\Delta t$ reflects statistical fluctuations in the observed background rate, as well as the presence of individual events of varying quality. The dashed line labelled ' $90 \%$ Sensitivity' shows the upper limit that would have been obtained with exactly the mean expected background. The event at $30 \mathrm{~s}$ (event 1 ) is consistent with background and believed to be a cosmic-ray air shower.

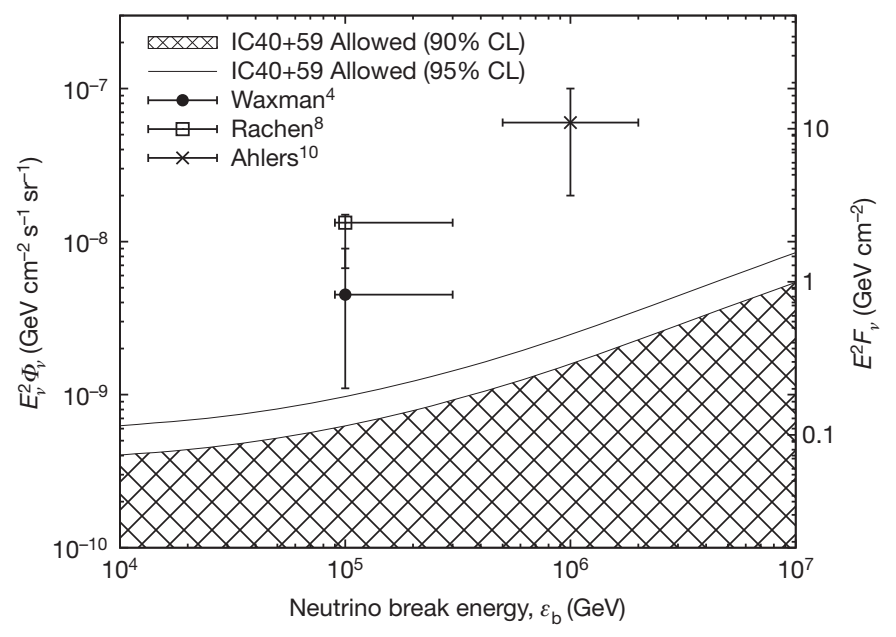

Figure 3 Compatibility of some neutrino flux predictions based on cosmic ray production in GRBs with observations. The cross-hatched area ('IC40+59 Allowed 90\% CL') shows the $90 \%$ confidence allowed values of the neutrino flux (vertical axes, as in Fig. 1) versus the neutrino break energy $\left(\varepsilon_{\mathrm{b}}\right)$ in comparison to model predictions with estimated uncertainties (points); the solid line labelled 'IC50+59 Allowed 95\% CL' shows the upper bound of the $95 \%$ confidence allowed region. Data were taken from the model-independent analysis from the time window corresponding to the median duration of the GRBs in our catalogue $(|\Delta t|=28 \mathrm{~s})$. Spectra are represented here as broken power laws $\left(\Phi_{v} \cdot\left\{E^{-1} / \varepsilon_{\mathrm{b}}, E<\varepsilon_{\mathrm{b}} ; E^{-2}, E>\varepsilon_{\mathrm{b}}\right\}\right)$ with a break energy $\varepsilon_{\mathrm{b}}$ corresponding to the $\Delta$ resonance for $p-\gamma$ interactions in the frame of the shock. The muon flux in IceCube is dominated by neutrinos with energies around the first break $\left(\varepsilon_{\mathrm{b}}\right)$. As such, the upper break, due to synchrotron losses of $\pi^{+}$, has been neglected here, as its presence or absence does not contribute significantly to the muon flux and thus does not have a significant effect on the presented limits. $\varepsilon_{\mathrm{b}}$ is related to the bulk Lorentz factor $\Gamma\left(\varepsilon_{\mathrm{b}} \propto \Gamma^{2}\right)$; all of the models shown assume $\Gamma \approx 300$. The value of $\Gamma$ corresponding to $10^{7} \mathrm{GeV}$ is $>1,000$ for all models. Vertical axes are related to the accelerated proton flux by the modeldependent constant of proportionality $f_{\pi}$. For models assuming a neutrondecay origin of cosmic rays (ref. 8 and ref. 10) $f_{\pi}$ is independent of $\Gamma$; for others (ref. 4) $f_{\pi} \propto \Gamma^{-4}$. Error bars on model predictions are approximate and were taken either from the original papers, where included ${ }^{10}$, or from the bestavailable source in the literature ${ }^{15}$ otherwise. The errors are due to uncertainties in $f_{\pi}$ and in fits to the cosmic-ray spectrum. Waxman-Bahcall ${ }^{4}$ (circle) and Rachen ${ }^{8}$ (box) fluxes were calculated using a cosmic-ray density of $(1.5-3) \times 10^{44} \mathrm{erg} \mathrm{Mpc}^{-3} \mathrm{yr}^{-1}$, with $3 \times 10^{44}$ the central value ${ }^{20}$. The Ahlers ${ }^{10}$ model is shown with a cross. CL, confidence level.

producing neutrinos at proton-photon $(p-\gamma)$ interactions in internal shocks. The remaining parameter spaces available to each model therefore have similar characteristics: either a low density of highenergy protons, below that required to explain the cosmic rays, or a low efficiency of neutrino production.

In the GRB fireball, protons are believed to be accelerated stochastically in collisions of internal shocks in the expanding GRB. The neutrino flux is proportional to the rate of $p-\gamma$ interactions, and so to the proton content of the burst by a model-dependent factor. Assuming a model-dependent proton ejection efficiency, the proton content can in turn be related to the measured flux of high-energy cosmic rays if GRBs are the cosmic-ray sources. Limits on the neutrino flux for cosmic-ray-normalized models are shown in Fig. 3; each model prediction has been normalized to a value consistent with the observed ultra-high-energy cosmic-ray flux. The proton density can also be expressed as a fraction of the observed burst energy, directly limiting the average proton content of the bursts in our catalogue (Fig. 4).

An alternative is to reduce the neutrino production efficiency, for example by modifying the physics included in the predictions ${ }^{16,17}$ or by increasing the bulk Lorentz boost factor, $\Gamma$. Increasing $\Gamma$ increases the proton energy threshold for pion production in the observer frame, thereby reducing the neutrino flux owing to the lower proton density at higher energies. Astrophysical lower limits on $\Gamma$ are established by pair 


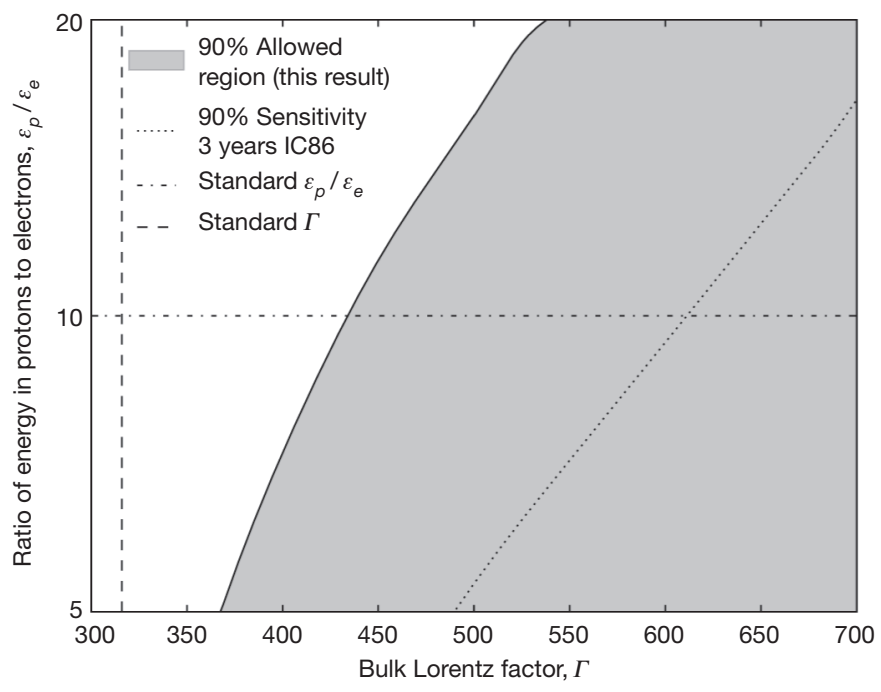

Figure $4 \mid$ Constraints on fireball parameters. The shaded region, based on the result of the model dependent analysis, shows the values of GRB energy in protons and the average fireball bulk Lorentz factor for modelled fireballs ${ }^{6,9}$ allowed by this result at the $90 \%$ confidence level. The dotted line indicates the values of the parameters to which the completed IceCube detector (IC86) is expected to be sensitive after 3 years of data. The standard values considered ${ }^{9}$ are shown as dashed-dotted and dashed lines and are excluded by this analysis. Note that the quantities shown here are model-dependent.

production arguments ${ }^{9}$, but the upper limit is less clear. Although it is possible that $\Gamma$ may take values of up to 1,000 in some unusual bursts, the average value is probably lower (usually assumed ${ }^{6,9}$ to be around $300)$ and the non-thermal $\gamma$-ray spectra from the bursts set a weak constraint that $\Gamma \lesssim 2,000$ (ref. 18). For all considered models, with uniform fixed proton content, very high average values of $\Gamma$ are required to be compatible with our limits (Figs 3,4).

In the case of models where cosmic rays escape from the GRB fireball as neutrons ${ }^{8,10}$, the neutrons and neutrinos are created in the same $p-\gamma$ interactions, directly relating the cosmic-ray and neutrino fluxes and removing many uncertainties in the flux calculation. In these models, $\Gamma$ also sets the threshold energy for production of cosmic rays. The requirement that the extragalactic cosmic rays be produced in GRBs therefore does set a strong upper limit on $\Gamma$ : increasing it beyond $\sim 3,000$ causes the proton flux from GRBs to disagree with the measured cosmic-ray flux above $4 \times 10^{18} \mathrm{eV}$, where extragalactic cosmic rays are believed to be dominant. Limits on $\Gamma$ in neutron-origin models from this analysis ( $\gtrsim 2000$, Fig. 3 ) are very close to this point, and as a result all such models - in which all extragalactic cosmic rays are emitted from GRBs as neutrons-are now largely ruled out.

Although the precise constraints are model-dependent, the general conclusion is the same for all the versions of fireball phenomenology we have considered here: either the proton density in GRB fireballs is substantially below the level required to explain the highest-energy cosmic rays or the physics in GRB shocks is significantly different from that included in current models. In either case, our current theories of cosmic-ray and neutrino production in GRBs will need to be revisited.

\section{Received 6 January; accepted 8 March 2012.}

1. Waxman, E. Cosmological gamma-ray bursts and the highest energy cosmic rays. Phys. Rev. Lett. 75, 386-389 (1995).

2. Vietri, M. The acceleration of ultra-high-energy cosmic rays in gamma-ray bursts. Astrophys. J. 453, 883-889 (1995)

3. Milgrom, M. \& Usov, V. Possible association of ultra-high-energy cosmic-ray events with strong gamma-ray bursts. Astrophys. J. 449, L37 (1995).

4. Waxman, E. \& Bahcall, J. High energy neutrinos from cosmological gamma-ray burst fireballs. Phys. Rev. Lett. 78, 2292-2295 (1997)

5. Avrorin, A. V. et al. Search for neutrinos from gamma-ray bursts with the Baika neutrino telescope NT200. Astron. Lett. 37, 692-698 (2011).

6. Abbasi, R. et al. Search for muon neutrinos from gamma-ray bursts with the IceCube neutrino telescope. Astrophys. J. 710, 346-359 (2010).
7. Abbasi, R. et al. Limits on neutrino emission from gamma-ray bursts with the 40 string IceCube detector. Phys. Rev. Lett. 106, 141101 (2011).

8. Rachen, J. P. \& Mészáros, P. in Fourth Huntsville Gamma-Ray Burst Symposium (eds Meegan, C. A., Preece, R. D. \& Koshut, T. M.) 776-780 (American Institute of Physics Conference Proceedings Vol. 428, 1998)

9. Guetta, D., Hooper, D., Alvarez-Muñiz, J., Halzen, F. \& Reuveni, E. Neutrinos from individual gamma-ray bursts in the BATSE catalog. Astropart. Phys. 20, 429-455 (2004).

10. Ahlers, M., Gonzalez-Garcia, M. C. \& Halzen, F. GRBs on probation: testing the UHE CR paradigm with IceCube. Astropart. Phys. 35, 87-94 (2011).

11. Becker, J. K. High-energy neutrinos in the context of multimessenger astrophysics. Phys. Rep. 458, 173-246 (2008)

12. Abbasi, R. et al. The IceCube data acquisition system: signal capture, digitization, and timestamping. Nucl. Instrum. Methods Phys. Res. A 601, 294-316 (2009).

13. Ahrens, J. et al. Muon track reconstruction and data selection techniques in AMANDA. Nucl. Instrum. Methods Phys. Res. A 524, 169-194 (2004).

14. GCN: The Gamma-ray Coordinates Network. http://gcn.gsfc.nasa.gov

15. Guetta, D., Spada, M. \& Waxman, E. On the neutrino flux from gamma-ray bursts Astrophys. J. 559, 101-109 (2001)

16. Baerwald, P., Hümmer, S. \& Winter, W. Systematics in aggregated neutrino fluxes and flavor ratios from gamma-ray bursts. Astropart. Phys. 35, 508-529 (2012).

17. Hümmer, S., Baerwald, P. \& Winter, W. Neutrino emission from gamma-ray burst fireballs, revised. Preprint at http://ArXiv.org/abs/1112.1076 (2011).

18. Mészáros, P. Gamma-ray bursts. Rep. Prog. Phys. 69, 2259-2321 (2006).

19. Becker, J. K. Stamatikos, M., Halzen, F. \& Rhode, W. Coincident GRB neutrino flux predictions: implications for experimental UHE neutrino physics. Astropart. Phys. 25, 118-128 (2006)

20. Waxman, E. Astrophysical sources of high energy neutrinos. Nucl. Phys. B Proc., Suppl. 118, 353-362 (2003).

21. Feldman, G. J. \& Cousins, R. D. Unified approach to the classical statistical analysis of small signals. Phys. Rev. D 57, 3873-3889 (1998).

Supplementary Information is linked to the online version of the paper at www.nature.com/nature.

Acknowledgements We acknowledge support from the following agencies: US NSF, Office of Polar Programs, and US NSF, Physics Division; University of Wisconsin Alumn Research Foundation; the GLOW and OSG grids; US DOE, NERSCC; the LONI grid; NSERC, Canada; Swedish Research Council, Swedish Polar Research Secretariat, SNIC, K. and A. Wallenberg Foundation, Sweden; German Ministry for Education and Research, Deutsche Forschungsgemeinschaft; Research Department of Plasmas with Complex Interactions (Bochum), Germany; FSR, FWO Odysseus, IWT, BELSPO, Belgium; University of Oxford, UK; Marsden Fund, New Zealand; Australian Research Council; JSPS, Japan; SNSF, Switzerland. J.P.R was supported by the Capes Foundation, Brazil; N.W. by the NSF GRFP. We thank S. Hümmer, E. Waxman and W. Winter for discussions.

Author Contributions The IceCube observatory was designed and constructed by the IceCube Collaboration and the IceCube Project. It is operated by the IceCube Collaboration, who set science goals. Data processing and calibration, Monte Carlo simulations of the detector and of theoretical models, and data analyses were performed by a large number of IceCube members who also discussed and approved the scientific results. This manuscript was written by P.R. and N.W. and subjected to an internal collaboration-wide review process. All authors approved the final version of the manuscript.

Author Information Reprints and permissions information is available at www.nature.com/reprints. The authors declare no competing financial interests. Readers are welcome to comment on the online version of this article at www.nature.com/nature. Correspondence and requests for materials should be addressed to N.W. (nwhitehorn@icecube.wisc.edu) or to P.R. (redlpete@icecube.umd.edu).

IceCube Collaboration R Abbasi ${ }^{1}$ Y Abdou ${ }^{2}$ T. Abu-Zayyad ${ }^{3}$ M. Ackermann ${ }^{4}$, Adams $^{5}$, J. A. Aguilar ${ }^{6}$, M. Ahlers ${ }^{1}$, D. Altmann', K. Andeen ${ }^{1}$, J. Auffenberg ${ }^{1}$, X. Bai ${ }^{8,9}$, M Baker $^{1}$, S. W. Barwick ${ }^{10}$, R. Bay ${ }^{11}$, J. L. Bazo Alba ${ }^{4}$, K. Beattie ${ }^{12}$, J. J. Beatty ${ }^{13,14}$, S. Bechet $^{15}$, J.K. Becker ${ }^{16}$, K.-H. Becker ${ }^{17}$, M. Bell ${ }^{18}$, M. L. Benabderrahmane ${ }^{4}$, S. BenZvi ${ }^{1}$ J. Berdermann ${ }^{4}$,P. Berghaus ${ }^{8}$, D. Berley ${ }^{19}$, E. Bernardini ${ }^{4}$, D. Bertrand ${ }^{15}$, D. Z. Besson ${ }^{20}$ D. Bindig ${ }^{17}$, M. Bissok ${ }^{7}$, E. Blaufuss ${ }^{19}$, J. Blumenthal' ${ }^{7}$, D. J. Boersma ${ }^{7}$, C. Bohm²1 ${ }^{21}$ D. Bose $^{22}$, S. Böser ${ }^{23}$, O. Botner ${ }^{24}$, L. Brayeur ${ }^{22}$, A. M. Brown ${ }^{5}$, S. Buitink ${ }^{22}$, K. S

Caballero-Mora ${ }^{18}$, M. Carson ${ }^{2}$, M. Casier ${ }^{22}$, D. Chirkin ${ }^{1}$, B. Christy ${ }^{19}$, F. Clevermann $^{25}, \mathrm{~S}$ Cohen $^{26}$, C. Colnard ${ }^{27}$, D. F. Cowen ${ }^{18,28}$, A. H. Cruz Silva ${ }^{4}$, M. V. D'Agostino ${ }^{11}$, M. Danninger $^{21}$, J. Daughhetee ${ }^{29}$, J. C. Davis ${ }^{13}$, C. De Clercq ${ }^{22}$, T. Degner ${ }^{23}$, F. Descamps ${ }^{2}$ P. Desiati ${ }^{1}$ G. de Vries-Uiterweerd ${ }^{2}$, T. DeYoung ${ }^{18}$ J. C. Díaz-Vélez ${ }^{1}$ M. Dierckxsens ${ }^{15}$ J Dreyer ${ }^{16}$, J. P. Dumm ${ }^{1}$, M. Dunkman ${ }^{18}$, J. Eisch ${ }^{1}$, R. W. Ellsworth ${ }^{19}$, O. Engdegård ${ }^{24}$, S. Euler $^{7}$, P. A. Evenson ${ }^{8}$, O. Fadiran ${ }^{1}$, A. R. Fazely ${ }^{30}$, A. Fedynitch ${ }^{16}$, J. Feintzeig', T. Feusels ${ }^{2}$ K Filimonov ${ }^{11}$ C. Finley ${ }^{21}$ T. Fischer-Wasels ${ }^{17}$ S. Flis ${ }^{21}$ A. Franckowiak ${ }^{23}$ R Franke $^{4}$, T. K. Gaisser ${ }^{8}$, J. Gallagher ${ }^{31}$, L. Gerhardt ${ }^{11,12}$, L. Gladstone ${ }^{1}$, T. Glüsenkamp ${ }^{4}$ A. Goldschmidt ${ }^{12}$, J. A. Goodman ${ }^{19}$, D. Góra ${ }^{4}$, D. Grant ${ }^{32}$, T. Griesel ${ }^{33}$, A. Groß ${ }^{27}$, S. Grullon ${ }^{1}$, M. Gurtner ${ }^{17}$, C. Ha ${ }^{11,12}$, A. Haj Ismail ${ }^{2}$, A. Hallgren ${ }^{24}$, F. Halzen ${ }^{1}$, K. Han ${ }^{4}$, K. Hanson ${ }^{15}$, D. Heereman ${ }^{15}$ D. Heinen ${ }^{7}$, K. Helbing ${ }^{17}$, R. Hellauer ${ }^{19}$ S. Hickford ${ }^{5}$. G. C. Hill $^{34}$, K. D. Hoffman ${ }^{19}$, B. Hoffmann ${ }^{7}$, A. Homeier ${ }^{23}$, K. Hoshina ${ }^{1}$, W. Huelsnitz ${ }^{19,35}$, J.-P. Hül $\beta^{7}$, P. O. Hulth ${ }^{21}$, K. Hultqvist ${ }^{21}$, S. Hussain ${ }^{8}$, A. Ishihara ${ }^{36}$, E. Jacobi ${ }^{4}$, J. Jacobsen ${ }^{1}$, G. S. Japaridze ${ }^{37}$, H. Johansson ${ }^{21}$, A. Kappes ${ }^{38}$, T. Karg ${ }^{17}$, A. Karle ${ }^{1}$, J.Kiryluk ${ }^{39}$, F. Kislat ${ }^{4}$, S R. Klein ${ }^{11,12}$, J.-H. Köhne ${ }^{25}$, G. Kohnen ${ }^{40}$, H. Kolanoski ${ }^{38}$, L. Köpke ${ }^{33}$, S. Kopper ${ }^{17}$, D. J. 
RESEARCH LETTER

Koskinen $^{18}$, M. Kowalski ${ }^{23}$, T. Kowarik ${ }^{33}$, M. Krasberg ${ }^{1}$, G. Kroll ${ }^{33}$, J. Kunnen ${ }^{22}$, N. Kurahashi ${ }^{1}$, T. Kuwabara ${ }^{8}$, M. Labare ${ }^{22}$, K. maihem ${ }^{7}$, H. Landsman ${ }^{1}$, M. J. Larson ${ }^{18}$, R. Laver $^{4}$, J. Lünemann ${ }^{33}$, J. Madsen ${ }^{3}$, A. Marotta ${ }^{15}$, R. Maruyama ${ }^{1}$, K. Mas $^{36}$, H. S. Metis $^{12}$, K. Meager ${ }^{19}$, M. Merck ${ }^{1}$, P. Mészáros ${ }^{18,28}$, T. Meres ${ }^{15}$, S. Miarecki ${ }^{11,12}$, E. Middell $^{4}$, N. Milker ${ }^{25}$, J. Miller ${ }^{24}$,T. Montaruli, $61,{ }^{6}$, R. Morse ${ }^{1}$, S. M. Movie ${ }^{28}$, R. Nahnhauer ${ }^{4}$ J.W. Nam ${ }^{10}$, U. Naumann ${ }^{17}$, S. C. Nowicki ${ }^{32}$, D. R. Nygren ${ }^{12}$, S. Odrowski ${ }^{27}$, A. Olives ${ }^{19}$ M. Olive ${ }^{16}$, A. O'Murchadha ${ }^{1}$, S. Panknin ${ }^{23}$, L. Paul ${ }^{7}$, C. Pérez de los Hers ${ }^{24}$, A. Piegsa ${ }^{33}$, D. Pieloth ${ }^{25}$, J. Posset ${ }^{17}$, P. B. Price ${ }^{11}$, G. T. Przybylski ${ }^{12}$, K. Rawlings ${ }^{42}$, P. Red ${ }^{19}$, E. Resconi $^{27,43}$,W. Rhode ${ }^{25}$, M. Ribordy ${ }^{26}$, M. Richman ${ }^{19}$, B. Riedel $^{1}$, A. Rizzio ${ }^{22}$, J. P.

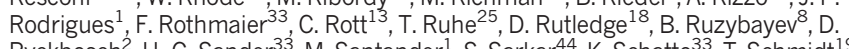
Ryckbosch $^{2}$, H.-G. Sander ${ }^{33}$, M. Santander ${ }^{1}$, S. Sarkar ${ }^{44}$, K. Schatto ${ }^{33}$, T. Schmidt $^{19}$, S. Schöneberg $^{16}$, A. Schönwald ${ }^{4}$, A. Schukraft ${ }^{7}$, L. Schultz ${ }^{23}$, A. Schultes $^{17}$, O. Schulz ${ }^{27,43}$, M. Schunck , D. Seckel ${ }^{8}$, B. Semburg ${ }^{17}$, S. H. Se o ${ }^{21}$, Y. Sestayo ${ }^{27}$, S. Seunarine ${ }^{45}, A$. Silvestri ${ }^{10}$, M. W. E. Smith ${ }^{18}$, G. M. Spiczak ${ }^{3}$, C. Spiering ${ }^{4}$, M. Stamatikos ${ }^{13,46}$ T. Stane $^{8}$,

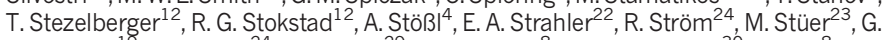

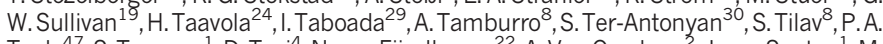
Tole $^{47}$, S. Toscano ${ }^{1}$, D. Toss ${ }^{4}$, N. van Eijndhoven ${ }^{22}$, A. Van Overlook ${ }^{2}$, J. van Santen ${ }^{1}$, M. Veering', M. Vouge ${ }^{23}$, C. Walk ${ }^{21}$, T. Waldenmaier ${ }^{38}$, M. Wallraff ${ }^{7}$, M. Walter ${ }^{4}$, R. Wassermann $^{18}$, Ch. Weaver ${ }^{1}$, C. Went ${ }^{1}$, S. Westerhoff ${ }^{1}$, N. Whitehorn ${ }^{1}$, K. Weber ${ }^{33}$, C. H. Wiebusch $^{7}$, D. R. Williams ${ }^{4}$, R. Wischnewski ${ }^{4}, H$. Kissing ${ }^{19}$, M. Wolf ${ }^{21}, T$. R. Wood ${ }^{32}, K$. Woschnagg ${ }^{11}$, C. Xu ${ }^{8}$, D. L. Xu ${ }^{47}$, X. W. Xu ${ }^{30}$, J. P. Yanez ${ }^{4}$, G. 'Yogh ${ }^{10}$, S. Yoshida ${ }^{36}$, P.' Zarzhitsky ${ }^{47}$ \& M. Lo ll ${ }^{21}$

${ }^{1}$ Department of Physics, University of Wisconsin, Madison, Wisconsin 53706, USA. ${ }^{2}$ Department of Physics and Astronomy, University of Gent, B-9000 Gent, Belgium. ${ }^{3}$ Department of Physics, University of Wisconsin, River Falls, Wisconsin 54022, USA. ${ }^{4}$ DESY, D-15735 Zeuthen, Germany. ${ }^{5}$ Department of Physics and Astronomy, University of Canterbury, Private Bag 4800, Christchurch, New Zealand. ${ }^{6}$ Département de physique nucléaire et corpusculaire, Université de Genève, $\mathrm{CH}-1211$ Genève, Switzerland. 'III. Physikalisches Institute, RWTH Aachen University, D-52056 Aachen, Germany. ${ }^{8}$ Bartol Research Institute and Department of Physics and Astronomy, University of Delaware, Newark, Delaware 19716, USA. ${ }^{9}$ Department of Physics, South Dakota School of Mines and Technology, Rapid City, South Dakota 57701, USA. ${ }^{10}$ Department of Physics and Astronomy, University of California, Irvine, California 92697, USA. ${ }^{11}$ Department of Physics, University of California, Berkeley, California 94720, USA. ${ }^{12}$ Lawrence Berkeley National Laboratory, Berkeley, California 94720, USA. ${ }^{13}$ Department of Physics and
Center for Cosmology and Astro-Particle Physics, Ohio State University, Columbus, Ohio 43210, USA. ${ }^{14}$ Department of Astronomy, Ohio State University, Columbus, Ohio 43210, USA. ${ }^{15}$ Université Litre de Bruxelles, Science Faculty CP230, B-1050 Brussels, Belgium. ${ }^{16}$ Fakultät für Physic \& Astronomic, Ruhr-Universität Bochum, D-44780 Bochum, Germany. ${ }^{17}$ Department of Physics, University of Wuppertal, D-42119 Wuppertal, Germany. ${ }^{18}$ Department of Physics, Pennsylvania State University, University Park, Pennsylvania 16802, USA. ${ }^{19}$ Department of Physics, University of Maryland, College Park, Maryland 20742, USA. ${ }^{20}$ Department of Physics and Astronomy, University of Kansas, Lawrence, Kansas 66045, USA. ${ }^{21}$ Oskar Klein Centre and Department of Physics, Stockholm University, SE-10691 Stockholm, Sweden. ${ }^{22}$ Vrije Universiteit Brussel, Dienst ELEM, B-1050 Brussels, Belgium. ${ }^{23}$ Physikalisches Institute, Universität Bonn, Nussallee 12, D-53115 Bonn, Germany. ${ }^{24}$ Department of Physics and Astronomy, Uppsala University, Box 516, S-75120 Uppsala, Sweden. ${ }^{25}$ Department of Physics, TU Dortmund University, D-44221 Dortmund, Germany. ${ }^{26}$ Laboratory for High Energy Physics, École Polytechnique Fédérale, $\mathrm{CH}-1015$ Lausanne, Switzerland. ${ }^{27}$ Max-Planck-Institut für Kernphysik, D-69177 Heidelberg, Germany. ${ }^{28}$ Department of Astronomy and Astrophysics, Pennsylvania State University, University Park, Pennsylvania 16802, USA. ${ }^{29}$ School of Physics and Center for Relativistic Astrophysics, Georgia Institute of Technology, Atlanta, Georgia 30332, USA. ${ }^{30}$ Department of Physics, Southern University, Baton Rouge, Louisiana 70813, USA. ${ }^{31}$ Department of Astronomy, University of Wisconsin, Madison, Wisconsin 53706, USA. ${ }^{32}$ Department of Physics, University of Alberta, Edmonton, Alberta, Canada T6G 2G7. ${ }^{33}$ Institute of Physics, University of Mainz, Staudinger We 7,D-55099 Mainz, Germany. ${ }^{34}$ School of Chemistry \& Physics, University of Adelaide, Adelaide 5005, South Australia, Australia. ${ }^{35}$ Los Alamo National Laboratory, Los Alamos, New Mexico 87545, USA. ${ }^{36}$ Department of Physics, Chiba University, Chiba 263-8522, Japan. ${ }^{37}$ CTSPS, Clark-Atlanta University, Atlanta, Georgia 30314, USA. ${ }^{38}$ Institut fur Physic, Humboldt-Universität zu Berlin, D-12489 Berlin, Germany.

${ }^{39}$ Department of Physics and Astronomy, Stony Brook University, Stony Brook, New York 11794-3800, USA. ${ }^{40}$ Université de Mons, 7000 Mons, Belgium. ${ }^{41}$ Dipartimento di Fisica, Sezione INFN, I-70126, Bari, Italy. ${ }^{42}$ Department of Physics and Astronomy, University of Alaska Anchorage, 3211 Providence Drive, Anchorage, Alaska 99508, USA. ${ }^{43}$ Technische Universität München, D-85748 Garching, Germany. ${ }^{44}$ Department of Physics, University of Oxford, 1 Keble Road, Oxford OX 1 3NP, UK. ${ }^{45}$ Department of Physics, University of the West Indies, Cave Hill Campus, Bridgetown BB 1 1000, Barbados. ${ }^{46}$ NASA Goddard Space Flight Center, Greenbelt, Maryland 20771, USA. ${ }^{47}$ Department of Physics and Astronomy, University of Alabama, Tuscaloosa, Alabama 35487, USA.

354 | NATURE | VOL 484 | 19 APRIL 2012

(C2012 Macmillan Publishers Limited. All rights reserved 